\title{
Importance of Shear in the bcc-to-hcp Transformation in Iron
}

\author{
Kyle J. Caspersen, ${ }^{1}$ Adrian Lew, ${ }^{2}$ Michael Ortiz, ${ }^{3}$ and Emily A. Carter ${ }^{1}$ \\ ${ }^{1}$ Department of Chemistry and Biochemistry, University of California, Los Angeles, California 90095-1569, USA \\ ${ }^{2}$ Department of Mechanical Engineering, Stanford University, Stanford, California 94305-4040, USA \\ ${ }^{3}$ Graduate School of Aeronautics, California Institute of Technology, Pasadena, California 91125, USA
}

(Received 16 March 2004; published 10 September 2004)

\begin{abstract}
Iron shows a pressure-induced martensitic phase transformation from the ground state ferromagnetic bcc phase to a nonmagnetic hcp phase at $\approx 13 \mathrm{GPa}$. The exact transformation pressure (TP) and pathway are not known. Here we present a multiscale model containing a quantum-mechanics-based multiwell energy function accounting for the bcc and hcp phases of Fe and a construction of kinematically compatible and equilibrated mixed phases. This model suggests that shear stresses have a significant influence on the bcc $\leftrightarrow$ hcp transformation. In particular, the presence of modest shear accounts for the scatter in measured TPs. The formation of mixed phases also provides an explanation for the observed hysteresis in TP.
\end{abstract}

DOI: 10.1103/PhysRevLett.93.115501

Pressure-driven phase transformations are ubiquitous and important for understanding the mechanical response of materials. In particular, the ground state crystal structure of Fe, ferromagnetic body-centered cubic (bcc), undergoes a pressure-induced martensitic phase transformation to a hexagonally close-packed (hcp) structure at $\approx 13 \mathrm{GPa}$. The measured transformation pressure (TP) varies greatly [1-3]. Attempts to simulate this transformation via quantum mechanics [4-8], primarily density functional theory (DFT), have focused on relative phase stability, where the TP is approximated by the Gibbs construction of drawing the line of common tangent between equations of state of the pure bcc and hcp phases. Another DFT model [9] mapped out the energetics of a constrained transformation path between the pure phases, similar to earlier work on $\mathrm{Ba}$ [10]. None of these were able to explain the large range of measured TPs. Here, we present a multiscale model containing a first-principles DFT-based multiwell energy function accounting for bcc and hcp Fe and a construction of kinematically compatible and equilibrated mixed phases (laminates) to represent the complicated microstructures often observed in experiments.

We confine our attention to transformations occurring at $0 \mathrm{~K}$ and therefore the governing principle is energy minimization. In particular, the formation of microstructure is driven purely by energetics. We assume the behavior of the material to be nonlinear elastic with energy density $W(\mathbf{F})$, where $\boldsymbol{F}$ is the local deformation gradient [11]. The function $W(\mathbf{F})$ must be invariant under rigidbody rotations, and therefore it must be of the form $W(\mathbf{C})$, where $\boldsymbol{C}=\boldsymbol{F}^{\mathbf{T}} \boldsymbol{F}$ and is the right Cauchy-Green deformation tensor [11]. In addition, if $G \subset S O(3)$ is the symmetry group of the crystal in its reference configuration, then we must have $W(\boldsymbol{F G})=W(\mathbf{F})$ for all $\mathbf{G}$ in $\boldsymbol{G}$.

We connect with first-principles DFT via the CauchyBorn hypothesis [12], which assumes that the crystal lattice is locally uniformly deformed according to the
PACS numbers: $61.50 . \mathrm{Ks}, 61.72 .-\mathrm{y}, 62.50 .+\mathrm{p}, 64.70 . \mathrm{Kb}$

deformation gradient $\mathbf{F}$. This hypothesis has been widely used in the mathematical analysis of martensites [13]. In principle, the Cauchy-Born hypothesis enables $W(\boldsymbol{C})$ to be calculated via 3D periodic DFT. However, the expense of converged first-principles calculations for Fe preclude an on-the-fly evaluation of $W(\boldsymbol{C})$. Additionally, a tabulated form of $W(\mathbf{C})$ is not feasible due to the fact that $\mathbf{C}$ is 6 dimensional. Instead, we consider a special form of $W(\boldsymbol{C})$, constructed below. The objective of this construction is to obtain a multiwell $W(\boldsymbol{C})$ with minima at symmetry-related deformations (variants) of bcc and hep iron. All information used in the construction of $W(\boldsymbol{C})$ is obtained from first principles.

We begin by considering the kinematics of this martensitic transformation. The particular transformation path considered is the well-known Burgers path [14]. Here the hcp lattice is obtained by first applying a shear deformation to a bcc $\{110\}$ plane, consisting of an elongation and compression along perpendicular directions, followed by a shuffle of the deformed $\{110\}$ planes. We define the untransformed bcc variant as the identity $\boldsymbol{I}$. The formation of an hep variant from $\boldsymbol{I}$ is represented by the matrix

$$
\boldsymbol{U}=\left(\begin{array}{ccc}
\frac{3}{4 \sqrt{2}}+\frac{\alpha}{2} & -\frac{3}{4 \sqrt{2}}+\frac{\alpha}{2} & 0 \\
-\frac{3}{4 \sqrt{2}}+\frac{\alpha}{2} & \frac{3}{4 \sqrt{2}}+\frac{\alpha}{2} & 0 \\
0 & 0 & \frac{\sqrt{3}}{2}
\end{array}\right) .
$$

Here $c / a=\sqrt{8 / 3} \alpha$. There exist six $\{110\}$ planes in the bcc lattice, resulting in six possible independent hcp variants $\boldsymbol{U} \boldsymbol{G}$, where $\boldsymbol{G}$ is an element of the bcc point group. Each hcp variant can transform back to a bcc variant by simply reversing the shuffle and the shear deformation $\boldsymbol{U} \boldsymbol{G}$ along an hcp $\{0001\}$ plane. From the six hep variants obtained thus far, reverse transformations along multiple $\{0001\}$ planes produces 12 additional bec variants $\boldsymbol{G}^{-1} \boldsymbol{U}^{-1} \boldsymbol{H} \boldsymbol{U} \boldsymbol{G}$, where $\boldsymbol{H}$ is in the point group of hcp. Additional bcc and hcp variants could be gener- 
ated via further transformations, however we confine ourselves to the 19 identified thus far.

The specific form of the energy density assumed is $W(\boldsymbol{C})=\min _{i=0, \ldots, 18} W^{i}(\boldsymbol{C})$, where

$$
W^{i}(\boldsymbol{C})=W_{0}^{i}(V)+\frac{1}{2}\left[\boldsymbol{C}-\boldsymbol{C}^{i}(V)\right] \mathbb{C}^{\mathbf{i}}(V)\left[\boldsymbol{C}-\boldsymbol{C}^{i}(V)\right] .
$$

The index $i$ enumerates the 19 variants described above; the volume $V=V_{\mathrm{bcc}} \sqrt{\operatorname{det} \mathbf{C}}$ and $V_{\mathrm{bcc}}$ is the volume of the undeformed bcc variant; $C^{i}(V)$ is the deformation of the $i$ th variant with volume $V$ under hydrostatic pressure conditions; $W_{0}^{i}(V) \equiv W\left[\boldsymbol{C}^{i}(V)\right]$ is the equation of state of the $i$ th variant, where by symmetry only two are unique, corresponding to the bcc and hcp phases; $\mathbb{C}^{\mathbf{i}}(V)$ are the elastic moduli, defined as $\mathbb{C}^{i}(V)=\partial^{2} W^{i} / \partial \boldsymbol{C}^{2}$ evaluated with $\boldsymbol{C}=\boldsymbol{C}^{i}(V)$ and at constant $V$. Note the elastic constants depend on the specific volume, and correspond to those at the deformation $C^{i}(V)$. For bcc, $\boldsymbol{C}^{i}(V)$ is an exclusively volumetric deformation, but for hcp, these account for the optimal $c / a$ ratio as a function of $V$, and therefore contain some shear deformation. This small dependence on shear is all that is needed, since, to a first approximation, the variants are considered to be close to their undistorted configuration. Consequently, Eq. (2) is a Taylor series expansion of $W^{i}$ through second order. The linear term is missing by definition of $\boldsymbol{C}^{i}(V)$.

We use first-principles DFT to calculate $\boldsymbol{C}^{i}(V)$ (specifically the optimal $c / a$ ratio for hcp as a function of $V$ ), $W_{0}^{i}(V)$, and $\mathbb{C}^{i}(V)$ in Eq. (2). Spin-polarized Kohn-Sham DFT calculations are performed within the VASP code [15], where the generalized gradient approximation [16] is employed to describe electron exchange and correlation. To accurately recover the magnetic and structural properties of both bcc and hcp Fe, the all-electron projector augmented wave method [17] is used. Because of the high precision needed to calculate elastic constants, a large $(24 \times 24 \times 24)$ Monkhorst-Pack k-point grid for the two atom cell and a $500 \mathrm{eV}$ kinetic energy cutoff for the planewave basis set is required.

The bcc equilibrium volume, bulk modulus, and magnetic moment for $\mathrm{Fe}$ are calculated to be $11.43 \AA$, $172 \mathrm{GPa}$, and $2.21 \mu_{B}$, respectively, which coincide well with experimental values of $11.70 \AA, 172 \mathrm{GPa}$ [18], and $2.22 \mu_{B}$ [19]. Additionally, the calculated equilibrium bcc $\mathrm{Fe}$ elastic constants, $c_{11}=271 \mathrm{GPa}, c_{12}=$ $145 \mathrm{GPa}$, and $c_{44}=101 \mathrm{GPa}$, are in fair agreement with experimental values, $c_{11}=243 \mathrm{GPa}, \quad c_{12}=$ $138 \mathrm{GPa}$, and $c_{44}=122 \mathrm{GPa}$ [18]. We find the equilibrium hcp energy to be $64.0 \mathrm{meV} /$ ion above bcc. We predict the hcp equilibrium volume, bulk modulus, and $c / a$ ratio to be $10.25 \AA^{3}, 293 \mathrm{GPa}$, and 1.58 , respectively. Although little experimental data exist for hcp, our results agree with previous first-principles calculations [20]. Experimental hcp elastic constants are not available. The actual elastic constant data used in Eq. (2) are shown in Fig. 1. The equations of state $W_{0}^{i}(V)$ and the minimum energy Cauchy-Green deformation tensors $C^{i}(V)$ are tabulated for use upon calculation by DFT (not shown). This completes the definition of the multiwell energy density $W(\boldsymbol{C})$ for $\mathrm{Fe}$.

Suppose now that the material undergoes a macroscopic deformation $\overline{\boldsymbol{F}}$. Since the above energy density has a multiwell structure, the minimum energy generally is not attained for a uniform deformation. Instead, the material adopts a fine microstructure consisting of a combination of variants. Unfortunately, at present no method exists for determining arbitrary optimal microstructures. Instead, we consider a special class of microstructures known as sequential laminates (e. g., [21,22]). Such sequential laminates are often observed experimentally $[23,24]$ for martensites.

A simple laminate is a compatible and mechanically equilibrated combination of two phases arranged in alternating layers. A sequential laminate is a laminate of laminates spanning a hierarchy of length scales, such as shown in the insets in Fig. 2. The rank of a laminate equals the number of levels of lamination. Each layer in the laminate undergoes a uniform deformation. The deformations of adjacent layers are constrained by compatibility, i.e., no sliding or gaps between the layers may occur as a result of the deformation. In addition, the average deformation of the laminate must equal the prescribed macroscopic deformation $\overline{\boldsymbol{F}}$. The computational problem then concerns the determination of the lamination layout, geometry, and deformation that minimizes the energy of the crystal under the compatibility and average deformation constraints. The compatibility constraint precludes direct optimization of the variant volume fractions so that the simple Gibbs construction does not apply [25]. The
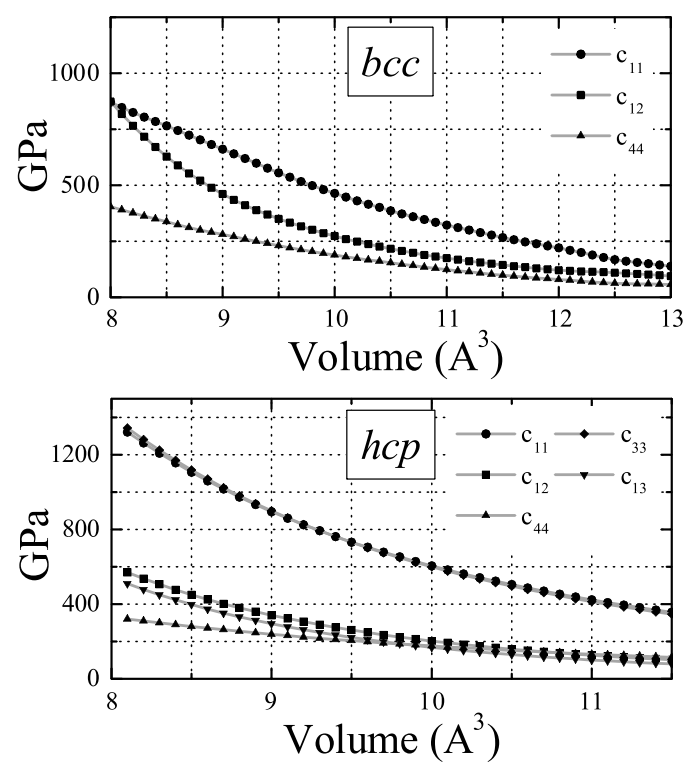

FIG. 1. Calculated bcc and hcp elastic constants as a function of volume. The $c_{11}$ and $c_{33}$ hcp elastic constants are almost identical throughout the volume range. 


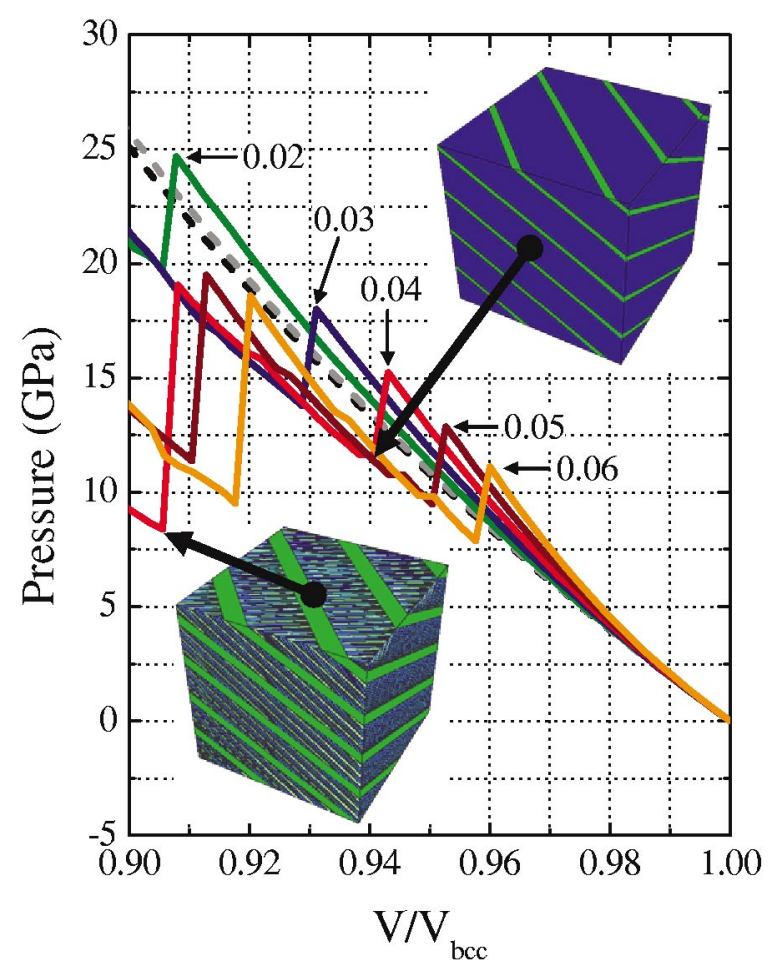

FIG. 2 (color). Pressure vs volume curves for increasing amounts of shear. The black and gray dashed curves correspond to $\epsilon_{f}=0$ and 0.01 , respectively; for all other curves the corresponding $\epsilon_{f}$ values are indicated explicitly. The discontinuities in the curves occur as a result of laminate formation. The insets are examples of predicted microstructure for the case in which $\epsilon_{f}=0.04$. The top represents a rank two laminate formed immediately after the transformation onset; the microstructure appears to be a rank one laminate, due to very thin layers within the hcp (green) phase that cannot be seen. The bottom inset shows the microstructure after an additional transformation, which is a rank four laminate.

specific algorithm used equilibrate the laminate and optimize its layout is given in [26].

To investigate the effect of shear on the transformation pressure, we consider the macroscopic affine deformation

$$
\overline{\boldsymbol{F}}_{\mathrm{S}}(\delta)=(1-\delta) \boldsymbol{I}+\delta\left(\begin{array}{ccc}
\lambda & \boldsymbol{\epsilon}_{f} & 0 \\
\boldsymbol{\epsilon}_{f} & \lambda & 0 \\
0 & 0 & \lambda
\end{array}\right)
$$

where $\delta$ ranges from 0 to 1 and $\lambda$ is chosen such that $\lambda\left(\lambda^{2}-\epsilon_{f}^{2}\right)=V_{f} / V_{\mathrm{bcc}} . V_{f}$ and $\epsilon_{f}$ are the final volume and final shear, respectively. All components of $\overline{\boldsymbol{F}}_{\mathrm{S}}$ refer to the cubic axes of undeformed bcc. This defines a oneparameter family of combined shear and volume deformations.

Figure 2 displays the pressure vs volume curves for a number of values of $\epsilon_{f}$, while the insets are examples of laminated microstructures. All data in Fig. 2 are generated with $V_{f}=0.89 V_{\text {bcc }}$.

Figure 2 shows shear is required for phase transformation to occur in this volume range. The discontinuities in the $P-V$ curves that appear for $\epsilon_{f} \geq 0.02$ correspond to the formation of laminates. The onset of these discontinuities depends sensitively on the amount of shear applied to the sample, with the onset appearing earlier in the compression sequence as shear increases. Specifically, a range of $\epsilon_{f}$ from 0.02 to 0.06 produces TPs of 11-25 GPa, which is in agreement with the range of TPs observed experimentally. This illustrates the sensitivity of the TP to small shears, suggesting that the variability in measured TPs may indeed be due to the different shear states that exist in experiments, e.g., in diamond anvil cell powder diffraction studies [1-3].

The laminates shown in Fig. 2 correspond to the structures formed immediately after drops in the $P-V$ curve for $\epsilon_{f}=0.04$. The upper laminate is of rank 2, while the lower laminate is of rank 4 . The volume fraction, orientation, and deformation of each of the variants in the laminate evolve with increasing macroscopic deformation. This complex microstructure provides an explicit physical model of the mixed phases commonly discussed by experimentalists. In particular, we see that a modest amount of shear gives rise to a broad range of pressures over which the material is neither pure bcc nor pure hcp. This provides a plausible explanation for the ambiguities at phase boundaries in measured phase diagrams and the discrepancies between various measurements [1].

To further investigate the properties of this martensitic transformation, we employ the volume-conserving deformation $\overline{\boldsymbol{F}}_{\mathrm{M}}(V, \delta)=\beta\left[(1-\delta) \boldsymbol{F}_{\mathrm{bcc}}(V)+\delta \boldsymbol{F}_{\mathrm{hcp}}(V)\right]$, where $\beta$ is chosen such that $\operatorname{det} \overline{\boldsymbol{F}}_{\mathrm{M}}(V, \delta)=V / V_{\mathrm{bcc}}$. Here $\quad \boldsymbol{F}_{\mathrm{bcc}}(V)=\left(V / V_{\mathrm{bcc}}\right)^{1 / 3} \boldsymbol{I}$ and $\boldsymbol{F}_{\mathrm{hcp}}(V)=$ $\left(V / V_{\text {bcc }}\right)^{1 / 3}[\operatorname{det} \boldsymbol{U}(V)]^{-1 / 3} \boldsymbol{U}(V)$ (see Eq. (1)) are affine deformations that produce specific bcc and hcp variants, respectively, at a volume $V$. We consider $V / V_{\mathrm{bcc}}$ ranging from 0.86 to 1.02 and $\delta$ ranging from 0 to 1 .

Figure 3 displays the energy $E(V)$ per ion, the pressure $P$, and the fraction $\chi$ of the hcp phase for the $\delta$ that minimizes the energy at a particular $V . E(V)$ varies smoothly throughout the volume range, and we see that the energy is lowered with respect to that of the individual phases. This stabilization is due to the formation of the mixed phase microstructure. The Gibbs construction is also shown for comparison in Fig. 3. This construction, which we term "perfect mixing," corresponds to mixing variants without imposing compatibility constraints and delivers a lower bound on the energy. In addition to compatibility constraints, our restriction on the number of allowed variants further frustrates the system. As a result, the system cannot laminate arbitrarily, effectively reducing the accessible configuration space, building in frustration. It is this frustration that causes the upward bowing in the $E-V$ curve in Fig. 3.

We also observe a lag in conversion from bcc-to-hcp, exhibited by an overshoot in the compression required to produce pure hcp compared to the hcp equilibrium volume. The transformation onset is at $V \approx 10.8 \AA^{3}$, much 


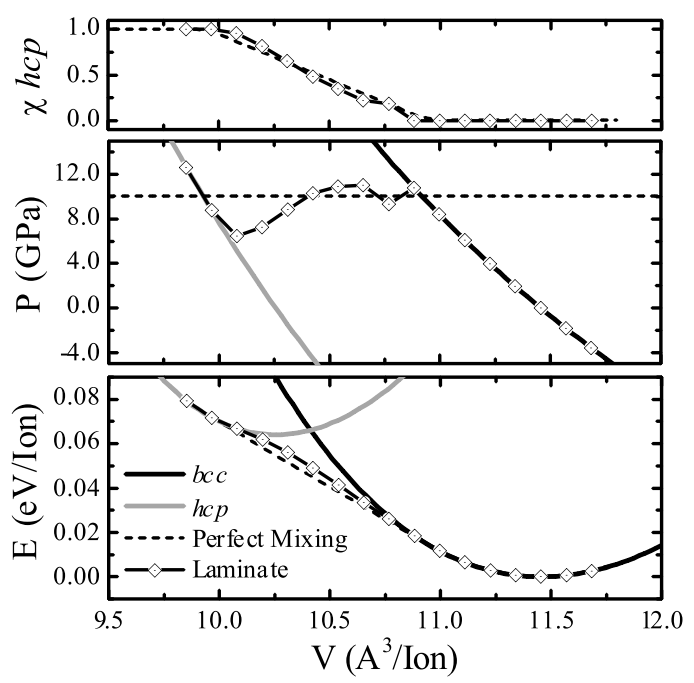

FIG. 3. Fraction of hcp $\chi$, pressure $P$, and energy $E(V)$ per atom, as a function of volume $V$ for the path $\boldsymbol{F}_{M}(V)=$ $\min _{\delta} W\left[\boldsymbol{F}_{M}(V, \delta)\right]$.

lower than $V_{\mathrm{bcc}}$. Full hep conversion is achieved only after $V \approx 10.0 \AA^{3}$, well below $V_{\text {hcp. }}$. This overshoot exists for the perfect mixing case as well; it is simply a consequence of hcp being less stable than bcc at zero pressure.

We also see a pressure drop during the transformation, corresponding to the bowing in the $E-V$ curve. This pressure drop implies that as the bcc phase is compressed, the bcc-to-hcp transformation occurs at $\approx 10.5 \mathrm{GPa}$, whereas when the hcp phase is decompressed, the hcp to bcc transformation occurs at $\approx 5.1 \mathrm{GPa}$. This suggests that pressure hysteresis may be simply due to kinematic constraints rather than kinetics. Additionally, the hysteresis width of $\approx 5 \mathrm{GPa}$ is consistent with measured hysteresis widths of, e.g., 6.2 GPa [27].

In conclusion, shear may be required to initiate the bccto-hcp transformation in Fe, as shear tends to stabilize the hcp phase. We predict that the transformation occurs at larger volumes and lower applied pressures with increasing shear, via the formation of a complex microstructure consisting of mixed phases. This may account for the ambiguities in measured phase diagrams and transformation pressures. We find that compatibility between the variants frustrates the system and causes the $P-V$ curve to be nonmonotonic. This behavior in turn may be responsible for the pressure hysteresis observed in the bcc to hcp transformation. While we have focused on Fe here, we expect these findings to apply qualitatively to all martensitic materials.

We close by pointing out strengths and limitations of our model. The multiscale model presented here is able to simulate structures at the micron scale, unlike molecular dynamics simulations. An additional advantage of our approach is that arbitrary deformations can be accounted for, not necessarily limited to volumetric deformations. This opens up greatly the richness of the phenomena that can be explored. Moreover, the approach is entirely predictive, since all input data come from first principles, with no calibration or fitting of the model to experiment. Lastly, the model currently does not yet account for nucleation, interfacial energies and kinetics, finite temperature, $f c c$ variants, or plasticity, but these effects can [26] and will be included in future work.

Support from the DOE through Caltech's ASCI/ASAP Center for the Simulation of the Dynamic Response of Materials and from the Office of Naval Research is gratefully acknowledged. Dr. Matt Fago is the primary author of the lamination code employed in this work.

[1] N. Von Bargen and R. Boehler, High Press. Res. 6, 133 (1990).

[2] J. P. Rueff et al., Phys. Rev. B 60, 14510 (1999).

[3] E. Huang et al., J. Geophys. Res. 92, 8129 (1987).

[4] L. Stixrude et al., Phys. Rev. B 50, 6442 (1994).

[5] E. G. Moroni and T. Jarlborg, Europhys. Lett. 33, 223 (1996).

[6] R. E. Cohen et al., Phys. Rev. B 56, 8575 (1997).

[7] L. Vočadlo et al., Faraday Discuss. 106, 205 (1997).

[8] P. Söderlind et al., Phys. Rev. B 53, 14063 (1995).

[9] M. Ekman et al., Phys. Rev. B 58, 5296 (1998).

[10] Y. Chen et al., Phys. Rev. B 37, 283 (1988).

[11] J. E. Marsden and T. J. R. Hughes, Mathematical Foundations of Elasticity (Dover Publications, New York, 1994).

[12] J. L. Ericksen, The Cauchy and Born Hypothesis for Crystals, in Phase Transformations and Material Instabilities in Solids (Academic Press, New York, 1984).

[13] K. Bhattacharya, Microstructure of Martensite: Why it Forms and How it Gives Rise to the Shape-memory Effect (Oxford University Press, Oxford, England, 2003).

[14] W. G. Burgers, Physica (Amsterdam) 1, 561 (1934).

[15] G. Kresse and J. Furthmüller, Phys. Rev. B 54, 11169 (1996).

[16] J. Perdew et al., Phys. Rev. B 46, 6671 (1992).

[17] P. E. Blöchl, Phys. Rev. B 50, 17953 (1994).

[18] G. Simmons and H. Wang, Single Crystal Elastic Constants and Calculated Aggregate Properties: A Handbook (MIT Press, Cambridge, MA, 1971).

[19] N.W. Ashcroft and N. D. Mermin, Solid State Physics (Saunders College Publishers, Philadelphia, 1976).

[20] G. Steinle-Neumann et al., Phys. Rev. B 60, 791 (1999).

[21] R. V. Kohn and G. Strang, Commun. Pure Appl. Math. 39, 113 (1986).

[22] J. Ball and R. D. James, Z. Angew. Math. Mech. 76, 389 (1996).

[23] H. G. Bowden and P. M. Kelly, Acta Metall. Mater. 15, 1489 (1967).

[24] C. Chu and R. D. James, J. Phys. IV (Colloque) 5, 143 (1995).

[25] R. V. Kohn, Continuum Mech. Thermodyn. 3, 193 (1991).

[26] S. Aubry, M. Fago, and M. Ortiz, Comput. Methods Appl. Mech. Eng. 192, 2823 (2003).

[27] R. D. Taylor et al., J. Appl. Phys. 69, 6126 (1991). 